Research Article

\title{
A Note on the Integration of Scalar Fourth-Order Ordinary Differential Equations with Four-Dimensional Symmetry Algebras
}

\author{
Said Waqas Shah ${ }^{(D)},{ }^{1}$ F. M. Mahomed, ${ }^{2,3}$ and H. Azad ${ }^{1}$ \\ ${ }^{1}$ Abdus Salam School of Mathematical Sciences GC University, Lahore. 68-B, New Muslim Town, Lahore 54600, Pakistan \\ ${ }^{2}$ DSI-NRF Centre of Excellence in Mathematical and Statistical Sciences, School of Computer Science and Applied Mathematics, \\ University of the Witwatersrand, Johannesburg 2050, Wits, South Africa \\ ${ }^{3}$ School of Mathematics and Statistics, University of New South Wales, Sydney NSW 2052, Australia \\ Correspondence should be addressed to Said Waqas Shah; waqas.shah@sms.edu.pk
}

Received 29 October 2020; Revised 25 January 2021; Accepted 2 March 2021; Published 19 March 2021

Academic Editor: Mariusz Michta

Copyright (c) 2021 Said Waqas Shah et al. This is an open access article distributed under the Creative Commons Attribution License, which permits unrestricted use, distribution, and reproduction in any medium, provided the original work is properly cited.

The complete integration of scalar fourth-order ODEs with four-dimensional symmetry algebras is performed by utilizing Lie's method which was invoked to integrate scalar second-order ODEs admitting two-dimensional symmetry algebras. We obtain a complete integration of all scalar fourth-order ODEs that possess four Lie point symmetries.

\section{Introduction}

Lie devised a method to integrate a scalar second-order ordinary differential equation (ODE) if it admits a twodimensional symmetry algebra by reducing the order of the equation twice and hence obtaining integrability by quadratures for the ODE (see $[1,2])$. Following the same method, Ibragimov and Nucci [3] gave an algorithmic procedure to integrate scalar third-order ODEs admitting three-dimensional symmetry algebras. In their paper, Cerquetelli et al. [4] classified scalar fourth-order ODEs admitting four-dimensional symmetry algebras by first presenting a classification of realizations of all four-dimensional Lie algebras in the plane. In their work, some cases were missing which were then completed by Fatima et al. in [5]. In [4], the authors had also introduced Lie's method for integrating fourth-order equations admitting four-dimensional symmetry algebras.

In this note, we extend these works to complete the integration of scalar fourth-order ODEs admitting fourdimensional symmetry algebras. First, we compare the realizations in the plane given in $[4,5]$ with that of the realizations given in González-López et al. [6] and show that they are compatible. For each case, we establish the required coordinate transformations. To denote a general four-dimensional algebra, we have used the notation in [5], and for the algebras in [6], the notation $(m, n)$, where $m$ is the type of algebra and $n$ is the dimension of the algebra which in our case is four, is utilized. We have then found all the scalar fourth-order ODEs with symmetry and have also used the classification given in $[4,5]$ whenever needed. Thereafter, we perform a complete integration of all scalar fourth-order ODEs possessing four symmetries. Note that, in [5], the authors only consider the Lie algebraic classification and not the integration of scalar fourth-order ODEs which admit four-dimensional symmetry algebras. Here, apart from the comparison of the realizations obtained in both $[4,5]$ with that of [6], we obtain a complete integration of all scalar fourth-order ODEs which admit four point symmetries. Also, we present applications of our results.

In Section 2, we present the integration of all scalar fourth-order ODEs that admit four Lie point symmetries. We utilise a three-dimensional ideal of the algebra to reduce the equation to order one which admits the quotient algebra 
and which is hence integrable. We present the main results in tabular form: first, the correspondence of the realizations utilized for fourth-order ODEs with that of González-López et al. [6] is given in Table 1. Then, in Table 2, the canonical forms of scalar fourth-order ODEs which possess 4 symmetries are presented. In Table 3 , invariant representation of 4th-order ODEs admitting 3-dimensional ideals is listed. This is followed by the integrability of 4 th-order ODEs with four symmetries in Table 4 . The main features of integrability are discussed thereafter. Section 3 deals with applications of scalar higher-order ODEs. These occur in practice in beam theory as well as in the reduction of partial differential equations (PDEs) which arise in several areas. A brief conclusion is provided with summary and related questions in the last section.

\section{Integration of Fourth-Order ODEs Admitting Four-Dimensional Algebras}

Let a scalar fourth-order ODE admit a four-dimensional symmetry algebra, say $\mathfrak{g}_{4}$. Such an algebra always has a three-dimensional ideal, say $\mathfrak{J}_{3}$. We use it to transform the fourth-order ODE into a first-order ODE in new coordinates which are precisely the differential invariants of $\mathfrak{I}_{3}$ of order $\leq 3$. This transformed first-order ODE admits $\mathfrak{g}_{4} / \mathfrak{\Im}_{3}$ and hence can be integrated easily [2]. The general form of solution of this first-order ODE in original coordinates is a scalar third-order ODE admitting $\mathfrak{I}_{3}$ which can be integrated further by Lie's method as performed in [3]. One notes that all the four-dimensional algebras are solvable except for two cases which are (8) and (9), and thus, the corresponding equations can be integrated easily by quadratures. We follow the same steps to integrate all possible scalar fourth-order ODEs possessing four-dimensional symmetry algebras. This is the reason that we give some of the cases in detail, and all the necessary information for other cases is divided into four tables in the following pages.

Remark 1. In Table 2, we have not stated the 4-dimensional algebras $(21,4)$ and $4 A_{1}$ of Table 1 for the reason that a 4thorder ODE admitting these can be mapped to linearizable ODEs of the same order by point transformations that admit fifth generators $\phi_{1}(x) \partial_{y}, \phi_{1}^{\prime \prime} \neq 0$, with $\phi_{1}$ solving the linearized ODE $y^{(4)}=\left(\phi^{(4)}(x) / \phi^{(2)}(x)\right) y^{\prime \prime}+H(x)\left(\phi^{(2)} y^{\prime \prime \prime}-\phi^{(3)}(x)\right.$ $\left.y^{\prime \prime}\right)\left(\phi_{1}\right.$ different from $\left.\phi\right)$ and $y \partial_{y}$ of a linear ODE, respectively. Since such ODEs must admit 5-dimensional algebras, we exclude these. In Tables $2-4$, we list the algebras of Table 1 as (1) to (23). Also, $a, b$ are constants and $H$ a function of its argument.

We present the salient features of integration of some of the cases as in the above tables according to the nomenclature utilized in the following tables below. The remaining cases in the tables are then easy to understand.

(1) $\left(2 A_{2}, 5\right): X_{1}=\partial_{x}, X_{2}=\partial_{y}, X_{3}=x \partial_{x}, X_{4}=y \partial_{y}$.

$(13,4)$ : with the same generators.
Fourth-order equation:

$$
y^{(4)}=\frac{y^{\prime \prime 3}}{y^{\prime 2}} H\left(\frac{y^{\prime} y^{\prime \prime \prime}}{y^{\prime \prime 2}}\right) .
$$

The three-dimensional ideal of the above algebra is generated by $X_{1}, X_{2}$, and $X_{3}$. Invariants of this threedimensional ideal of order $\leq 3$ are $u=y^{\prime \prime} y^{\prime-2}, v$ $=y^{\prime \prime \prime} y^{\prime^{-3}}$. Equation (1) in terms of $u$ and $v$ coordinates is

$$
\left(\frac{v}{u^{2}}-2\right) v^{\prime}+3 \frac{v}{u}=u H\left(\frac{v}{u^{2}}\right)
$$

Here, $X_{4}$ in terms of $u$ and $v$ coordinates is $-u \partial_{u}-2 v \partial_{v}$ which is admitted by equation (2). Defining new coordinates as $\bar{u}=\ln (1 / u), \bar{v}=\left(v / u^{2}\right), X_{4}$ is transformed into $\partial_{\bar{u}}$. Note that these coordinates can be found by solving the following system of linear first-order partial differential equations by the method of characteristics:

$$
\begin{aligned}
& -u \frac{\partial_{\bar{u}}}{\partial_{u}}-2 v \frac{\partial_{\bar{u}}}{\partial_{v}}=1, \\
& -u \frac{\partial_{\bar{v}}}{\partial_{u}}-2 v \frac{\partial_{\bar{v}}}{\partial_{v}}=0 .
\end{aligned}
$$

Equation (2) in terms of $\bar{u}$ and $\bar{v}$ coordinates is

$$
\bar{v}^{\prime}=G(\bar{v}),
$$

where $G(\bar{v}):=((H(\bar{v})-3 \bar{v}) /(2-\bar{v}))$ and the equation admits $\partial_{\bar{u}}$. Integrating this equation gives $\bar{v}=F\left(\bar{u}+c_{1}\right)$. This in terms of the original coordinates is a third-order equation admitting the three-dimensional algebra generated by $X_{1}, X_{2}$, and $X_{3}$ which can be integrated as in [3].

(9) $\quad A_{3,8} \oplus A_{1},\left(s \ell(2, I R) \oplus A_{1}, 8\right): \quad X_{1}=\partial_{y}, X_{2}=x \partial_{x}$ $+y \partial_{y}, X_{3}=2 x y \partial_{x}+y^{2} \partial_{y}, X_{4}=x \partial_{x}$.

$(19,4): X_{1}=\partial_{x}, X_{2}=2 x \partial_{x}+y \partial_{y}, X_{3}=x^{2} \partial_{x}+x y \partial_{y}$, $X_{4}=y \partial_{y}$.

Coordinate transformations: $\bar{x}=y, \bar{y}=x$.

Change in basis: $\overline{X_{1}}=X_{1}, \overline{X_{2}}=\left(X_{2} / 2\right)$, $\overline{X_{3}}=X_{3}, \overline{X_{4}}=X_{4}$.

Fourth-order equation:

$$
y^{(4)}=\frac{4}{3} \frac{y^{\prime \prime \prime 2}}{y^{\prime \prime}}+\frac{y^{\prime \prime 2}}{y} H\left(K_{1}\right)
$$

where $K_{1}=\left(y^{\prime \prime \prime 2} y / y^{\prime \prime 3}\right)+9\left(y^{\prime 2} / y y^{\prime \prime}\right)+6\left(y^{\prime} y^{\prime \prime \prime} / y^{\prime \prime 2}\right)$. The three-dimensional ideal: $X_{1}, X_{2}$, and $X_{3}$.

Invariants of order $\leq 3: u=y^{\prime \prime} y^{3}, v=y^{\prime \prime \prime} y^{5}+3 y^{\prime} y^{\prime \prime} y^{4}$. Equation (5) in terms of $u$ and $v$ coordinates is as follows:

$$
v^{\prime}=\frac{4}{3} \frac{v}{u}+\frac{u^{2}}{v}\left(3+H\left(\frac{v^{2}}{u^{3}}\right)\right)
$$

$X_{4}$ in terms of $u$ and $v$ coordinates: $4 u \partial_{u}+6 v \partial_{v}$. 
TABLE 1: Correspondence of the realizations in $[5,6]$.

\begin{tabular}{|c|c|c|c|}
\hline Algebra in [5] & Algebra in [6] & Transformed generators & Coordinate transformations \\
\hline$\left(2 A_{2}, 5\right)$ & $(13,4)$ & $X_{1}=\partial_{x}, X_{2}=\partial_{y}, X_{3}=x \partial_{x}, X_{4}=y \partial_{y}$ & Same generators \\
\hline$\left(2 A_{2}, 7\right)$ & $\begin{array}{c}(23,4) \\
r=2\end{array}$ & $X_{1}=e^{-x} \partial_{y}, X_{2}=\partial_{x}, X_{3}=\partial_{y}, X_{4}=y \partial_{y}$ & $\bar{x}=-\ln x, \bar{y}=y$ \\
\hline$A_{3,2} \oplus A_{1}$ & $\begin{array}{c}(22,4) \\
r=3\end{array}$ & $X_{1}=e^{-x} \partial_{y}, X_{2}=-x e^{-x} \partial_{y}, X_{3}=\partial_{x}, X_{4}=\partial_{y}$ & $\bar{x}=-x, \bar{y}=y e^{x}$ \\
\hline$A_{3,4}^{-1} \oplus A_{1}$ & $\begin{array}{c}(22,4) \\
r=3\end{array}$ & $X_{1}=e^{-x} \partial_{y}, X_{2}=e^{x} \partial_{y}, X_{3}=\partial_{x}, X_{4}=\partial_{y}$ & $\bar{x}=(\ln x / 2), \bar{y}=y|x|^{(-1 / 2)}$ \\
\hline$A_{3,4}^{a} \oplus A_{1}$ & $\begin{array}{c}(22,4) \\
r=3\end{array}$ & $X_{1}=e^{-x} \partial_{y}, X_{2}=e^{-a x} \partial_{y}, X_{3}=\partial_{x}, X_{4}=\partial_{y}$ & $\bar{x}=(\ln x /(1-a)), \bar{y}=y|x|^{(1 /(a-1))}$ \\
\hline$A_{3,5}^{0} \oplus A_{1}$ & $\begin{array}{c}(22,4) \\
r=3\end{array}$ & $X_{1}=\sin x \partial_{y}, X_{2}=\cos x \partial_{y}, X_{3}=\partial_{x}, X_{4}=\partial_{y}$ & $\bar{x}=\cot ^{-1} x, \bar{y}=\left(y /\left(\sqrt{1+x^{2}}\right)\right)$ \\
\hline$A_{3,5}^{b} \oplus A_{1}$ & $\begin{array}{c}(22,4) \\
r=3\end{array}$ & $X_{1}=e^{-b x} \sin x \partial_{y}, X_{2}=e^{-b x} \cos x \partial_{y}, X_{3}=\partial_{x}, X_{4}=\partial_{y}$ & $\bar{x}=\cot ^{-1} x, \bar{y}=\left(e^{-b \arctan x} /\left(\sqrt{1+x^{2}}\right)\right) y$ \\
\hline $\begin{array}{l}\left(s \ell(2, I R) \oplus A_{1}, 9\right) \\
\left(s \ell(2, I R) \oplus A_{1}, 8\right)\end{array}$ & $\begin{array}{l}(14,4) \\
(19,4)\end{array}$ & $\begin{array}{c}X_{1}=\partial_{x}, X_{2}=x \partial_{x}, X_{3}=x^{2} \partial_{x}, X_{4}=\partial_{y} \\
X_{1}=\partial_{x}, X_{2}=2 x \partial_{x}+y \partial_{y}, X_{3}=x^{2} \partial_{x}+x y \partial_{y}, X_{4}=y \partial_{y}\end{array}$ & $\begin{array}{c}\bar{x}=y, \bar{y}=x \\
\text { Scaling } X_{2} \text { by }(1 / 2) \text { only }\end{array}$ \\
\hline$A_{4,1}$ & $\begin{array}{c}(22,4) \\
r=3\end{array}$ & $X_{1}=\partial_{y}, X_{2}=x \partial_{y}, X_{3}=\left(x^{2} / 2\right) \partial_{y}, X_{4}=\partial_{x}$ & Same generators \\
\hline$A_{4,2}^{b}, b \neq 0,1$ & $\begin{array}{c}(22,4) \\
r=3\end{array}$ & $X_{1}=e^{-b x} \partial_{y}, X_{2}=e^{-x} \partial_{y}, X_{3}=-x e^{-x} \partial_{y}, X_{4}=\partial_{x}$ & $\bar{x}=(\ln x /(b-1)), \bar{y}=y|x|^{(b /(1-b))}$ \\
\hline$A_{4,3}$ & $\begin{array}{c}(22,4) \\
r=3\end{array}$ & $X_{1}=e^{-x} \partial_{y}, X_{2}=\partial_{y}, X_{3}=-x \partial_{y}, X_{4}=\partial_{x}$ & $\bar{x}=\ln x, \bar{y}=(y / x)$ \\
\hline$A_{4,4}$ & $\begin{array}{c}(22,4) \\
r=3\end{array}$ & $X_{1}=e^{-x} \partial_{y}, X_{2}=-x e^{-x} \partial_{y}, X_{3}=\left(x^{2} / 2\right) e^{-x} \partial_{y}, X_{4}=\partial_{x}$ & $\bar{x}=-x, \bar{y}=y e^{x}$ \\
\hline$A_{4,5}^{a, b, c}$ & $\begin{array}{c}(22,4) \\
r=3\end{array}$ & $X_{1}=e^{-a x} \partial_{y}, X_{2}=e^{-b x} \partial_{y}, X_{3}=e^{-x} \partial_{y}, X_{4}=\partial_{x}$ & $\bar{x}=x, \bar{y}=y e^{-a x}$ \\
\hline$A_{4,5}^{1,1,1}$ & $\begin{array}{c}(21,4) \\
r=2\end{array}$ & $X_{1}=\partial_{y}, X_{2}=x \partial_{y}, X_{3}=\phi(x) \partial_{y}, X_{4}=y \partial_{y}$ & $\bar{x}=\xi_{1}(x)$ and $\bar{y}=y$ \\
\hline$A_{4,6}^{a, b}, a>0$ & $\begin{array}{c}(22,4) \\
r=3\end{array}$ & $X_{1}=e^{-a x} \partial_{y}, X_{2}=e^{-b x} \cos x \partial_{y}, X_{3}=e^{-b x} \sin x \partial_{y}, X_{4}=\partial_{x}$ & $\bar{x}=x, \bar{y}=y e^{-a x}$ \\
\hline$A_{4,7}$ & $\begin{array}{c}(25,4) \\
\quad r=2\end{array}$ & $X_{1}=\partial_{y}, X_{2}=x \partial_{y}, X_{3}=-\partial x, X_{4}=x \partial x+\left(2 y+x^{2}\right) \partial y$ & $\bar{x}=x, \bar{y}=y+x^{2} \ln x^{\frac{3}{2}}$ \\
\hline$A_{4,8}^{b}, b=-1$ & $(24,4), \alpha=0$ & $X_{1}=\partial_{y}, X_{2}=\partial_{x}, X_{3}=x \partial_{y}, X_{4}=x \partial_{x}$ & Same generators \\
\hline $\begin{array}{l}A_{4,8}^{b},|b| \leq 1 \\
b \neq-1\end{array}$ & $\begin{array}{l}(24,4) \\
\alpha=b+1\end{array}$ & $X_{1}=\partial_{y}, X_{2}=\partial_{x}, X_{3}=x \partial_{y}, X_{4}=x \partial_{x}+(1+b) y \partial_{y}$ & Same generators \\
\hline$A_{4,8}^{b}, b=1$ & $\begin{array}{c}(24,4) \\
\alpha=2\end{array}$ & $X_{1}=\partial_{y}, X_{2}=\partial_{x}, X_{3}=x \partial_{y}, X_{4}=x \partial_{x}+2 y \partial_{y}$ & Same generators \\
\hline$A_{4,8}^{b}, b=0$ & $\begin{array}{c}(24,4) \\
\alpha=1\end{array}$ & $X_{1}=\partial_{y}, X_{2}=\partial_{x}, X_{3}=x \partial_{y}, X_{4}=x \partial_{x}+y \partial_{y}$ & Same generators \\
\hline$A_{4,8}^{b}, b \neq \pm 1$ & $\begin{array}{c}(24,4) \\
\alpha=((b+1) / b)\end{array}$ & $X_{1}=\partial_{y}, X_{2}=\partial_{x}, X_{3}=x \partial_{y}, X_{4}=x \partial_{x}+((1+b) / b) y \partial_{y}$ & Scaling $X_{4}$ by $(1 / b)$ \\
\hline$\left(A_{4,10}, 7\right)$ & $\begin{array}{c}(23,4) \\
r=2\end{array}$ & $X_{1}=\sin x \partial_{y}, X_{2}=\cos x \partial_{y}, X_{3}=y \partial_{y}, X_{4}=\partial_{x}$ & $\bar{x}=\cot ^{-1} x, \bar{y}=\left(y /\left(1+x^{2}\right)^{(1 / 2)}\right)$ \\
\hline$\left(A_{4,10}, 6^{*}\right)$ & $(4,4)$ & $X_{1}=\partial_{y}, X_{2}=\partial_{x}, X_{3}=x \partial_{x}+y \partial_{y}, X_{4}=-y \partial_{x}+x \partial_{y}$ & Same generators \\
\hline $4 A_{1}$ & $\begin{array}{c}(20,4) \\
r=3\end{array}$ & $X_{1}=\partial_{y}, X_{2}=x \partial_{y}, X_{3}=\xi_{2}(x) \partial_{y}, X_{4}=\xi_{3}(x) \partial_{y}$ & $\bar{x}=\xi_{1}(x)$ and $\bar{y}=y$ \\
\hline
\end{tabular}

TABLE 2: Representative 4th-order ODEs with 4 symmetries.

\begin{tabular}{|c|c|c|}
\hline Number & Generators of algebras in [6] & Corresponding fourth-order invariant equations \\
\hline (1) & $X_{1}=\partial_{x}, X_{2}=\partial_{y}, X_{3}=x \partial_{x}, X_{4}=y \partial_{y}$ & $y^{(4)}=\left(y^{\prime \prime 3} / y^{\prime 2}\right)\left(H\left(y^{\prime} y^{\prime \prime \prime}\right) / y^{\prime \prime} 2\right)$ \\
\hline (2) & $X_{1}=e^{-x} \partial_{y}, X_{2}=\partial_{x}, X_{3}=\partial_{y}, X_{4}=y \partial_{y}$ & $y^{(4)}=-y^{\prime \prime \prime}+\left(y^{\prime \prime}+y^{\prime \prime \prime}\right) H\left(\left(y^{\prime \prime}+y^{\prime \prime \prime}\right) /\left(y^{\prime}+y^{\prime \prime}\right)\right)$ \\
\hline (3) & $X_{1}=e^{-x} \partial_{y}, X_{2}=-x e^{-x} \partial_{y}, X_{3}=\partial_{x}, X_{4}=\partial_{y}$ & $y^{(4)}=-y^{\prime \prime \prime}+\left(y^{\prime \prime}+y^{\prime \prime \prime}\right) H\left(\left(y^{\prime \prime}+y^{\prime \prime \prime}\right) /\left(y^{\prime}+y^{\prime \prime}\right)\right)$ \\
\hline (4) & $X_{1}=e^{-x} \partial_{y}, X_{2}=e^{x} \partial_{y}, X_{3}=\partial_{x}, X_{4}=\partial_{y}$ & $y^{(4)}=y^{\prime \prime}+H\left(y^{\prime \prime \prime}-y^{\prime}\right)$ \\
\hline (5) & $X_{1}=e^{-x} \partial_{y}, X_{2}=e^{-a x} \partial_{y}, X_{3}=\partial_{x}, X_{4}=\partial_{y}$ & $y^{(4)}=-(a+1) y^{\prime \prime \prime}-a y^{\prime \prime}+H\left(y^{\prime \prime \prime \prime}+(a+1) y^{\prime \prime}+a y^{\prime}\right)$ \\
\hline (6) & $X_{1}=\sin x \partial_{y}, X_{2}=\cos x \partial_{y}, X_{3}=\partial_{x}, X_{4}=\partial_{y}$ & $y^{(4)}=-y^{\prime \prime}+H\left(y^{\prime \prime \prime}+y^{\prime}\right)$ \\
\hline (7) & $\begin{array}{c}X_{1}=e^{-b x} \sin x \partial_{y}, X_{2}=e^{-b x} \cos x \partial_{y} \\
X_{3}=\partial_{x}, X_{4}=\partial_{y}\end{array}$ & $\begin{array}{c}y^{(4)}=-2 b y^{\prime \prime \prime}-\left(b^{2}+1\right) y^{\prime \prime \prime}+H\left(K_{1}\right) \\
K_{1}=y^{\prime \prime \prime}+2 b y^{\prime \prime}+\left(b^{2}+1\right) y^{\prime}\end{array}$ \\
\hline (8) & $X_{1}=\partial_{x}, X_{2}=x \partial_{x}, X_{3}=x^{2} \partial_{x}, X_{4}=\partial_{y}$ & $\begin{aligned} y^{(4)}= & 3\left(y^{\prime \prime 3} / y^{\prime 2}\right)+6 K_{1} y^{\prime \prime} y^{\prime 2}+y^{\prime 4} H\left(K_{1}\right) \\
& K_{1}=y^{\prime \prime \prime} y^{\prime-3}-(3 / 2) y^{\prime \prime 2} y^{\prime-4}\end{aligned}$ \\
\hline
\end{tabular}


TABle 2: Continued.

\begin{tabular}{|c|c|c|}
\hline Nur & \multirow{5}{*}{$\begin{array}{c}\text { Generators of algebras in [6] } \\
X_{1}=\partial_{x}, X_{2}=2 x \partial_{x}+y \partial_{y} \\
X_{3}=x^{2} \partial_{x}+x y \partial_{y}, X_{4}=y \partial_{y} \\
X_{1}=\partial_{y}, X_{2}=x \partial_{y}, X_{3}=\left(x^{2} / 2\right) \partial_{y}, X_{4}=\partial_{x} \\
X_{1}=e^{-b x} \partial_{y}, X_{2}=e^{-x} \partial_{y}, X_{3}=-x e^{-x} \partial_{y}, X_{4}=\partial_{x} \\
X_{1}=e^{-x} \partial_{y}, X_{2}=\partial_{y}, X_{3}=-x \partial_{y}, X_{4}=\partial_{x} \\
X_{1}=e^{-x} \partial_{y}, X_{2}=-x e^{-x} \partial_{y} \\
X_{3}=\left(x^{2} / 2\right) e^{-x} \partial_{y}, X_{4}=\partial_{x}\end{array}$} & \multirow{16}{*}{$\begin{array}{c}\text { Corresponding fourth-order invariant equations } \\
y^{(4)}=(4 / 3)\left(y^{\prime \prime \prime} / y^{\prime \prime}\right)+\left(y^{\prime \prime 2} / y\right) H\left(K_{1}\right) \\
K_{1}=\left(y^{\prime \prime \prime} / y^{\prime \prime 2}\right)+9\left(y^{\prime 2} / y y^{\prime \prime}\right)+6\left(y^{\prime} y^{\prime \prime \prime} / y^{\prime \prime 2}\right) \\
y^{(4)}=H\left(y^{\prime \prime \prime}\right) \\
y^{(4)}=-(b+2) y^{\prime \prime \prime}-(2 b+1) y^{\prime \prime}-b y^{\prime}+H\left(K_{1}\right) \\
K_{1}=y^{\prime \prime \prime}+(2+b) y^{\prime \prime}+(2 b+1) y^{\prime}+b y \\
y^{(4)}=-y^{\prime \prime \prime}+H\left(y^{\prime \prime}+y^{\prime \prime \prime}\right) \\
y^{(4)}=y^{\prime \prime \prime} H\left(y^{\prime \prime \prime} e^{x}\right) \\
y^{(4)}=-(a+b+1) y^{\prime \prime \prime}-(a+b+a b) y^{\prime \prime}-a b y^{\prime}+H\left(K_{1}\right) \\
K_{1}=y^{\prime \prime \prime}+(a+b+1) y^{\prime \prime}+(a+b+a b) y^{\prime}+a b y \\
y^{(4)}=-(a+2 b) y^{\prime \prime \prime}-\left(b^{2}+2 a b+1\right) y^{\prime \prime}-a\left(b^{2}+1\right) y^{\prime}+H\left(K_{1}\right) \\
K_{1}=y^{\prime \prime \prime}+(a+2 b) y^{\prime \prime}+\left(b^{2}+2 a b+1\right) y^{\prime}+a\left(b^{2}+1\right) y \\
y^{(4)}=y^{\prime \prime \prime} H\left(y^{\prime \prime \prime} e^{y^{\prime \prime}}\right)\end{array}$} \\
\hline $\begin{array}{l}(9) \\
(10)\end{array}$ & & \\
\hline (11) & & \\
\hline (12) & & \\
\hline 13) & & \\
\hline (14) & $X_{1}=e^{-a x} \partial_{y}, X_{2}=e^{-b x} \partial_{y}, X_{3}=e^{-x} \partial_{y}, X_{4}=\partial_{x}$ & \\
\hline (15) & $\begin{array}{c}X_{1}=e^{-a x} \partial_{y}, X_{2}=e^{-b x} \cos x \partial_{y} \\
X_{3}=e^{-b x} \sin x \partial_{y}, X_{4}=\partial_{x}\end{array}$ & \\
\hline (16) & $\begin{array}{c}X_{1}=\partial_{y}, X_{2}=x \partial_{y} \\
X_{3}=-\partial x, X_{4}=x \partial x+\left(2 y+x^{2}\right) \partial y\end{array}$ & \\
\hline 17) & $X_{1}=\partial_{y}, X_{2}=\partial_{x}, X_{3}=x \partial_{y}, X_{4}=x \partial_{x}$ & \\
\hline 18) & $X_{1}=\partial_{y}, X_{2}=\partial_{x}, X_{3}=x \partial_{y}, X_{4}=x \partial_{x}+(1+b) y \partial_{y}$ & \\
\hline 19) & $X_{1}=\partial_{y}, X_{2}=\partial_{x}, X_{3}=x \partial_{y}, X_{4}=x \partial_{x}+2 y \partial_{y}$ & \\
\hline 20) & $X_{1}=\partial_{y}, X_{2}=\partial_{x}, X_{3}=x \partial_{y}, X_{4}=x \partial_{x}+y \partial_{y}$ & \\
\hline (21) & $\tau_{1}=\partial_{y}, X_{2}=\partial_{x}, X_{3}=x \partial_{y}, X_{4}=x \partial_{x}+((1+b) / b) y \partial_{y}$ & \\
\hline (22) & $X_{1}=\sin x \partial_{y}, X_{2}=\cos x \partial_{y}, X_{3}=y \partial_{y}, X_{4}=\partial_{x}$ & \\
\hline 23) & $X_{1}=\partial_{y}, X_{2}=\partial_{x}$ & \\
\hline & & \\
\hline
\end{tabular}

TABLE 3: Invariant representation of 4th-order ODEs admitting 3-dimensional ideals.

\begin{tabular}{|c|c|c|c|}
\hline Algebras [6] & 3-dimensional ideal & Invariants of order $\leq 3$ & Invariant equation \\
\hline $\begin{array}{l}(1) \\
(2)\end{array}$ & $\begin{array}{l}\left\langle X_{1}, X_{2}, X_{3}\right\rangle \\
\left\langle X_{1}, X_{2}, X_{3}\right\rangle\end{array}$ & $\begin{array}{c}u=y^{\prime \prime} y^{\prime^{-2}}, v=y^{\prime \prime \prime} y^{\prime^{-3}} \\
u=y^{\prime}+y^{\prime \prime}, v=y^{\prime \prime}+y^{\prime \prime \prime}\end{array}$ & $\begin{aligned}\left(\left(v / u^{2}\right)-2\right) v^{\prime} & +3(v / u)=u H\left(v / u^{2}\right) \\
v^{\prime} & =H(v / u)\end{aligned}$ \\
\hline (3) & $\left\langle X_{1}, X_{2}, X_{3}\right\rangle$ & $\begin{array}{l}u=y^{\prime \prime}+2 y^{\prime}+y \\
v=y^{\prime \prime \prime}+2 y^{\prime \prime}+y^{\prime}\end{array}$ & $v^{\prime}=H(v)$ \\
\hline (4) & $\left\langle X_{1}, X_{2}, X_{4}\right\rangle$ & $u=x, v=y^{\prime \prime \prime}-y^{\prime}$ & $v^{\prime}=H(v)$ \\
\hline (5) & $\left\langle X_{1}, X_{2}, X_{4}\right\rangle$ & $\begin{array}{c}u=x \\
v=y^{\prime \prime \prime}+(a+1) y^{\prime \prime}+a y^{\prime}\end{array}$ & $v^{\prime}=H(v)$ \\
\hline (6) & $\left\langle X_{1}, X_{2}, X_{4}\right\rangle$ & $u=x, v=y^{\prime \prime \prime}+y^{\prime}$ & $v^{\prime}=H(v)$ \\
\hline (7) & $\left\langle X_{1}, X_{2}, X_{4}\right\rangle$ & $\begin{array}{c}u=x \\
v=y^{\prime \prime \prime}+2 b y^{\prime \prime}+\left(b^{2}+1\right) y^{\prime}\end{array}$ & $v^{\prime}=H(v)$ \\
\hline $\begin{array}{l}(8) \\
(9) \\
(10)\end{array}$ & $\begin{array}{l}\left\langle X_{1}, X_{2}, X_{3}\right\rangle \\
\left\langle X_{1}, X_{2}, X_{3}\right\rangle \\
\left\langle X_{1}, X_{2}, X_{3}\right\rangle\end{array}$ & $\begin{array}{c}u=y, v=y^{\prime \prime \prime} y^{\prime-3}-(3 / 2) y^{\prime \prime 2} y^{\prime-4} \\
u=y^{\prime \prime} y^{3}, v=y^{\prime \prime \prime} y^{5}+3 y^{\prime} y^{\prime \prime} y^{4} \\
u=x, v=y^{\prime \prime \prime}\end{array}$ & $\begin{array}{c}v^{\prime}=H(v) \\
v^{\prime}=(4 / 3)(v / u)+\left(u^{2} / v\right)\left(3+H\left(v^{2} / u^{3}\right)\right) \\
v^{\prime}=H(v)\end{array}$ \\
\hline (11) & $\left\langle X_{1}, X_{2}, X_{3}\right\rangle$ & $\begin{array}{c}u=x \\
v=y^{\prime \prime \prime}+(2+b) y^{\prime \prime}+(2 b+1) y^{\prime}+b y\end{array}$ & $v^{\prime}=H(v)$ \\
\hline $\begin{array}{l}(12) \\
(13)\end{array}$ & $\begin{array}{l}\left\langle X_{1}, X_{2}, X_{3}\right\rangle \\
\left\langle X_{1}, X_{2}, X_{3}\right\rangle\end{array}$ & $\begin{array}{c}u=x, v=y^{\prime \prime \prime}+y^{\prime \prime} \\
u=x, v=y^{\prime \prime}\end{array}$ & $\begin{array}{c}v^{\prime}=H(v) \\
v^{\prime}=v H\left(v e^{u}\right)\end{array}$ \\
\hline (14) & $\left\langle X_{1}, X_{2}, X_{3}\right\rangle$ & $\begin{array}{c}u=x \\
v=y^{\prime \prime \prime}+(a+b+1) y^{\prime \prime}+ \\
(a+b+a b) y^{\prime}+a b y\end{array}$ & $v^{\prime}=H(v)$ \\
\hline (15) & $\left\langle X_{1}, X_{2}, X_{3}\right\rangle$ & $\begin{array}{c}u=x \\
v=y^{\prime \prime \prime}+(a+2 b) y^{\prime \prime}+ \\
\left(b^{2}+2 a b+1\right) y^{\prime}+a\left(b^{2}+1\right) y\end{array}$ & $v^{\prime}=H(v)$ \\
\hline (16) & $\left\langle X_{1}, X_{2}, X_{3}\right\rangle$ & $u=y^{\prime \prime}, v=y^{\prime \prime \prime}$ & $v^{\prime}=v H\left(v^{2} e^{u}\right)$ \\
\hline (17) & $\left\langle X_{1}, X_{2}, X_{3}\right\rangle$ & $u=y^{\prime \prime}, v=y^{\prime \prime \prime}$ & $v^{\prime}=v^{(1 / 3)} H\left(v^{-2} / u^{-3}\right)$ \\
\hline (18) & $\left\langle X_{1}, X_{2}, X_{3}\right\rangle$ & $u=y^{\prime \prime}, v=y_{\prime \prime \prime \prime}^{\prime \prime \prime}$ & $v^{\prime}=v^{(-1 /(b-2))} H\left(v^{b-1} / u^{b-2}\right)$ \\
\hline (19) & $\left\langle X_{1}, X_{2}, X_{3}\right\rangle$ & $u=y^{\prime \prime}, v=y^{\prime \prime \prime}$ & $v^{\prime}=v H(u)$ \\
\hline
\end{tabular}


TABle 3: Continued.

\begin{tabular}{lccc}
\hline Algebras [6] & 3-dimensional ideal & Invariants of order $\leq 3$ & Invariant equation \\
\hline$(20)$ & $\left\langle X_{1}, X_{2}, X_{3}\right\rangle$ & $u=y^{\prime \prime}, v=y^{\prime \prime \prime}$ & $v^{\prime}=v^{(1 / 2)} H\left(v^{-1} / u^{-2}\right)$ \\
$(21)$ & $\left\langle X_{1}, X_{2}, X_{3}\right\rangle$ & $u=y^{\prime \prime}, v=y^{\prime \prime \prime}$ & $v^{\prime}=v^{(-b /(1-2 b))} H\left(v^{((b-1) / b)} / u^{((b-2) / b)}\right)$ \\
$(22)$ & $\left\langle X_{1}, X_{2}, X_{3}\right\rangle$ & $u=y^{\prime \prime}+y, v=y^{\prime \prime \prime}+y^{\prime}$ & $v^{\prime}=H(v / u)$ \\
$(23)$ & $\left\langle X_{1}, X_{2}, X_{4}\right\rangle$ & $u=y^{\prime}, v=y^{\prime \prime \prime} y^{\prime \prime}$ & $v^{\prime}=-2 v^{2}+\left(\left(15 u^{2}+10 u K_{1}+H\left(K_{1}\right)\right) /\left(1+u^{2}\right)^{2}\right)$ \\
& & & $K_{1}=\left(1+u^{2}\right) v-3 u$ \\
\hline
\end{tabular}

TABLE 4: Integrability of 4th-order ODEs admitting 4 symmetries.

\begin{tabular}{|c|c|c|c|}
\hline Algebras. [6] & Symmetry & Canonical coordinates & Integrable equation \\
\hline (1) & $-u \partial_{u}-2 v \partial_{v}$ & $\bar{u}=\ln (1 / u), \bar{v}=\left(v / u^{2}\right)$ & $\begin{array}{c}\bar{v}^{\prime}=G(\bar{v}) \\
G(\bar{v}):=((H(\bar{v})-3 \bar{v}) /(2-\bar{v}))\end{array}$ \\
\hline (2) & $u \partial_{u}+v \partial_{v}$ & & $\bar{v}^{\prime}=H(\bar{v})$ \\
\hline (3) & $\partial_{u}$ & & $v^{\prime}=H(v)$ \\
\hline (4) & $\partial_{u}^{u}$ & & $v^{\prime}=H(v)$ \\
\hline (5) & $\partial_{u}^{u}$ & $\bar{u}=\ln u, \bar{v}=(v / u)$ & $v^{\prime}=H(v)$ \\
\hline (6) & $\partial_{u}$ & & $v^{\prime}=H(v)$ \\
\hline (7) & $\partial_{u}^{u}$ & & $v^{\prime}=H(v)$ \\
\hline (8) & $\partial_{u}^{u}$ & & $v^{\prime}=H(v)$ \\
\hline (9) & $4 u \partial_{u}+6 v \partial_{v}$ & & $\begin{array}{c}v^{\prime}=H(v) \\
G(\bar{v}):=(32 / 3) \bar{v}+8 H(\bar{v})+24\end{array}$ \\
\hline (10) & $\partial_{u}$ & $\bar{u}=(\ln u / 4)$ and $\bar{v}=\left(v^{2} / u^{3}\right)$ & $v^{\prime}=H(v)$ \\
\hline (11) & $\partial_{u}^{u}$ & & $v^{\prime}=H(v)$ \\
\hline (12) & $\partial_{u}^{u}$ & & $v^{\prime}=H(v)$ \\
\hline (13) & $-\partial_{u}+v \partial_{v}$ & & $\bar{v}^{\prime}=G(\bar{v})$ \\
\hline (14) & $\partial_{u}$ & $\bar{u}=-u$ and $\bar{v}=v e^{u}$ & $\begin{array}{c}G(\bar{v}):=-\bar{v} H(\bar{v}) \\
v^{\prime}=H(v)\end{array}$ \\
\hline (15) & $\partial_{u}^{u}$ & & $v^{\prime}=H(v)$ \\
\hline (16) & $2 \partial_{u}-v \partial_{v}$ & $\bar{u}=(u / 2)$ and $\bar{v}=v^{2} e^{u}$ & $\begin{aligned} \bar{v}^{\prime} & =G(\bar{v}) \\
G(\bar{v}): & =4 \bar{v} H(\bar{v})\end{aligned}$ \\
\hline (17) & $-2 u \partial_{u}-3 v \partial_{v}$ & $\bar{u}=-(1 / 2) \ln u, \bar{v}=\left(v^{-2} / u^{-3}\right)$ & $\begin{aligned} \bar{v}^{\prime} & =G(\bar{v}) \\
G(\bar{v}): & =4 \bar{v}^{2 / 3} H(\bar{v})\end{aligned}$ \\
\hline (18) & $(b-1) u \partial_{u}+(b-2) v \partial_{v}$ & $\bar{u}=(1 /(b-1)) \ln u, \bar{v}=\left(v^{b-1} / u^{b-2}\right)$ & $\begin{array}{c}\bar{v}^{\prime}=G(\bar{v}) \\
G(\bar{v}):=(b-1)^{2} \bar{v}^{((b-1) /(b-2))} H(\bar{v})\end{array}$ \\
\hline (19) & $-v \partial_{v}$ & $\bar{u}=u, \bar{v}=-\ln v$ & $\bar{v}^{\prime}=-H(\bar{u})$ \\
\hline$(20)$ & $-u \partial_{u}-2 v \partial_{v}$ & $\bar{u}=-\ln u, \bar{v}=\left(v^{-1} / u^{-2}\right)$ & $\begin{aligned} \bar{v}^{\prime} & =G(\bar{v}) \\
G(\bar{v}): & =\bar{v}^{1 / 2} H(\bar{v})\end{aligned}$ \\
\hline (21) & $((1-b) / b) u \partial_{u}+((1-2 b) / b) v \partial_{v}$ & $\bar{u}=(b /(1-b)) \ln u, \bar{v}=\left(v^{((b-1) / b)} / u^{((b-2) / b)}\right)$ & $\begin{array}{c}\bar{v}^{\prime}=G(\bar{v}) \\
G(\bar{v}):=\left(b^{2} /(1-b)^{2}\right) \bar{v}^{((1-b) /(1-2 b))} H(\bar{v})\end{array}$ \\
\hline (22) & $u \partial_{u}+v \partial_{v}$ & $\bar{u}=\ln u, \bar{v}=(v / u)$ & $\bar{v}^{\prime}=H(\bar{v})$ \\
\hline$(23)$ & $\left(1+u^{2}\right) \partial_{u}+(3-2 u v) \partial_{v}$ & $\bar{u}=\arctan u, \bar{v}=\left(1+u^{2}\right) v-3 u$ & $\begin{aligned} & \bar{v}^{\prime}=G(\bar{v}) \\
G(\bar{v}): & =H(\bar{v})-2 \bar{v}^{2}-3\end{aligned}$ \\
\hline
\end{tabular}

The coordinates which transform $X_{4}$ into $\partial_{\bar{u}}: \bar{u}=$ $(\ln u / 4)$ and $\bar{v}=\left(v^{2} / u^{3}\right)$.

Equation (6) in terms of $\bar{u}$ and $\bar{v}$ coordinates:

$$
\bar{v}^{\prime}=\frac{32}{3} \bar{v}+8 H(\bar{v})+24 .
$$

We define $G(\bar{v}):=(32 / 3) \bar{v}+8 H(\bar{v})+24$. Then, equation (7) becomes

$$
\bar{v}^{\prime}=G(\bar{v})
$$

Admitting $\partial_{\bar{u}}$, equation (8) can now easily be integrated.

(24) $\left(A_{4,10}, 6^{*}\right): \quad X_{1}=\partial_{y}, X_{2}=\partial_{x}, X_{3}=x \partial_{x}+y \partial_{y}$, $X_{4}=-y \partial_{x}+x \partial_{y}$.

$(4,4)$ : with the same generators.

Fourth-order equation:

$$
y^{(4)}=y^{\prime \prime 3}\left(\frac{15 y^{\prime 2}+10 y^{\prime} K_{1}+H\left(K_{1}\right)}{\left(1+y^{\prime 2}\right)^{2}}\right) \text {, }
$$


where $K_{1}=\left(\left(1+y^{\prime 2}\right) y^{\prime \prime \prime} / y^{\prime \prime 2}\right)-3 y^{\prime}$. The three-dimensional ideal of the above algebra is generated by $X_{1}, X_{2}$, and $X_{3}$. Invariants of this ideal of order $\leq 3$ are $u=y^{\prime}, v=y^{\prime \prime \prime} y^{\prime \prime}$. Equation (9) in terms of $u$ and $v$ coordinates is

$$
v^{\prime}=-2 v^{2}+\frac{15 u^{2}+10 u K_{1}+H\left(K_{1}\right)}{\left(1+u^{2}\right)^{2}}
$$

where $K_{1}=\left(1+u^{2}\right) v-3 u$. This equation admits $X_{4}$ which in terms of $u$ and $v$ coordinates is $\left(1+u^{2}\right) \partial_{u}+(3-2 u v) \partial_{v}$. Defining new coordinates as $\bar{u}=\arctan u, \bar{v}=\left(1+u^{2}\right) v-3 u, X_{4}$ transforms into $\partial_{\bar{u}}$. Equation (10) in terms of $\bar{u}$ and $\bar{v}$ coordinates then becomes

$$
\bar{v}^{\prime}=G(\bar{v})
$$

where $G(\bar{v}):=H(\bar{v})-2 \bar{v}^{2}-3$. This equation clearly admits $\partial_{\bar{u}}$. Integrating equation (11) gives $\bar{v}=F\left(\bar{u}+c_{1}\right)$ which in original coordinates is a third-order equation admitting the three-dimensional algebra generated by $X_{1}, X_{2}$, and $X_{3}$ and therefore can be integrated further. All the cases are given in Tables 1-4.

\section{Applications}

Fourth-order ODEs have many applications. They occur as reductions of PDEs in, for example, fluid mechanics and dynamic beam theory (see, e.g., [7-10]). Also, they appear in static beam theory [11].

The static Euler-Bernoulli beam ODE is of order 4 and has the following form:

$$
\frac{\mathrm{d}^{2}}{\mathrm{~d} x^{2}}\left(E I \frac{\mathrm{d}^{2} y}{\mathrm{~d} x^{2}}\right)=g .
$$

This ODE relates the static beam's deflection $y$ at some point $x$ with the applied load $g$ which can depend upon $x$ and $y$ and the derivatives of the deflection $y$. Here, the product $E I$ is the flexural rigidity and, in many engineering applications, is taken as constant.

Another form of a fourth-order ODE in beam-column theory is

$$
E I \frac{\mathrm{d}^{4} y}{\mathrm{~d} x^{4}}+P \frac{\mathrm{d}^{2} y}{\mathrm{~d} x^{2}}=f,
$$

in which $y$ is the deflection laterally and $f$ the intensity of the distributed load. Also, $P$ stands for the axial compressive force.

Interesting work on certain classes of the static beam model has been carried out in $[12,13]$ in which the authors utilise the Lie and Noether approaches for reductions and solutions.

Here, we show how the integration scheme obtained here for fourth-order ODEs with four symmetries can be used. If $E I$ is constant, then (12) is a general fourth-order ODE. It can be integrated by quadrature via the approach here, provided it has 4 symmetries. There are thus many cases as stated in Table 4. We mention two other examples below for each of (12) and (13).

(1) In (6) of Table 2 , we take $E I=1$ and $P=1$. Then, the fourth-order ODE becomes

$$
y^{(4)}+y^{\prime \prime}=H\left(y^{\prime}+y^{\prime \prime \prime}\right)
$$

Therefore, the intensity of the distributed load $f$ as in (13) is $H$. This ODE is integrable, as shown in Tables 3 and 4.

(2) If we take $E I=1$ in (12) and $g=y^{\prime \prime \prime}{ }^{2} H\left(y^{\prime \prime}\right)$, then it becomes

$$
y^{(4)}=y^{\prime \prime \prime 2} H\left(y^{\prime \prime}\right)
$$

This corresponds to (19) in Table 2 and is integrable by quadratures.

\section{Conclusion}

In this note, we firstly presented a reconciliation of the existing realizations utilized for the classification of scalar fourth-order ODEs that maximally admit four-dimensional Lie algebras. Then, we provided a complete integration algorithm for such equations thus completing the works $[4,5]$. The main results are concisely presented in Section 2, wherein the correspondence of the realizations of $[4,5]$ is given with those of [6]. Then, the scalar fourth-order equations which possess four symmetries are listed followed by their complete integrability.

In Section 3, we presented applications of the integration results to equations in beam theory and fluids mechanics. It is worthwhile mentioning that higher-order ODEs arise in several applications, including symmetry reductions of PDEs (see $[7,14,15]$ ), and various integration strategies are useful, not least the symmetry approach advanced here.

\section{Data Availability}

Mathematical models and proofs are described.

\section{Conflicts of Interest}

The authors declare that there are no conflicts of interest in this work.

\section{Acknowledgments}

FM thanks the NRF of South Africa for support.

\section{References}

[1] S. Lie, Vorlesungen über Differentialgeichungen mit bekannten infinitesimalen Transformationen, B.G. Teubner, Leipzig, Germany, 1892.

[2] N. K. Ibragimov, "Group analysis of ordinary differential equations and the invariance principle in mathematical physics (for the 150th anniversary of Sophus Lie)," Russian Mathematical Surveys, vol. 47, no. 4, pp. 89-156, 1992. 
[3] N. H. Ibragimov and M. C. Nucci, "Integration of third order ordinary differential equations by Lie's method: equations admitting three-dimensional Lie algebras," Lie Groups and Their Applications, vol. 1, pp. 49-64, 1994.

[4] T. Cerquetelli, N. Ciccoli, and M. C. Nucci, "Four dimensional Lie symmetry algebras and fourth order ordinary differential equations," Journal of Nonlinear Mathematical Physics, vol. 9, no. sup2, pp. 24-35, 2002.

[5] A. Fatima, M. Ayub, and F. M. Mahomed, "A note on fourdimensional symmetry algebras and fourth-order ordinary differential equations," Journal of Applied Mathematics, vol. 2013, no. 9, 2013.

[6] A. González-López, N. Kamran, P. J. Olver, and P. J. Olver, "Lie algebras of vector fields in the real plane," Proceedings of the London Mathematical Society, vol. s3-64, no. 2, pp. 339368, 1992.

[7] T. Aziz, F. M. Mahomed, and D. P. Mason, "A unified compatibility method for exact solutions of non-linear flow models of Newtonian and non-Newtonian fluids," International Journal of Non-linear Mechanics, vol. 78, pp. 142-155, 2016.

[8] A. H. Bokhari, F. M. Mahomed, and F. D. Zaman, "Invariant boundary value problems for a fourth-order dynamic EulerBernoulli beam equation," Journal of Mathematical Physics, vol. 53, no. 4, p. $043703,2012$.

[9] C. Wafo Soh, "Euler-Bernoulli beams from a symmetry standpoint-characterization of equivalent equations," Journal of Mathematical Analysis and Applications, vol. 345, pp. 387-395, 2008.

[10] E. Ozkaya and M. Pakdemirli, "Group-theoretic approach to axially accelerating beam problem," Acta Mechanica, vol. 155, pp. 111-123, 2002.

[11] A. E. H. Love, A Treatise on the Mathematical Theory of Elasticity, Dover, New York, NY, USA, 4 edition, 1944.

[12] A. H. Bokhari, F. M. Mahomed, and F. D. Zaman, "Symmetries and integrability of a fourth-order Euler-Bernoulli beam equation," Journal of Mathematical Physics, vol. 51, p. $053517,2010$.

[13] I. L. Freire, P. L. da Silva, and M. Torrisi, "Lie and Noether symmetries for a class of fourth-order Emden-Fowler equations," Journal of Physics A: Mathematical and Theoretical, vol. 46, no. 24, p. 245206, 2013.

[14] A. Isu Aliyu, M. Inc, A. Yusuf, and D. Beleanu, "Symmetry analysis, explicit solutions, and conservation laws of a sixthorder nonlinear ramani equation," Symmetry, vol. 10, no. 8, p. 341, 2018.

[15] A. Isu Aliyu, M. Inc, A. Yusuf, and D. Beleanu, "Invariant subspace and Lie symmetry analysis, exact solutions and conservation laws of a nonlinear reaction-diffusion murray equation arising in mathematical biology," Journal of Advanced Physics, vol. 7, no. 2, pp. 178-182, 2018. 